\title{
Multifunction Radar Concept for Through-Wall Surveillance
}

\author{
J. J. M. de Wit / W. L. van Rossum \\ Department of Radar Technology, TNO \\ The Hague, The Netherlands \\ jacco.dewit@tno.nl / \\ wim.vanrossum@tno.nl
}

\begin{abstract}
Radar systems suitable for stand-off mapping of building structures and through-wall surveillance allow covert reconnaissance of buildings and surveillance of people inside in support of e.g. police raids or search and rescue operations. In this paper the operational concept will be outlined for a multifunction radar system capable of stand-off building imaging and mapping, through-wall tracking of moving people, and blueforce tracking. These capabilities are illustrated with the aid of representative measurements.
\end{abstract}

Through-Wall Radar, FMCW Radar, Through-Wall Radar Tracking, Building Mapping

\section{INTRODUCTION}

Radar systems suitable for stand-off mapping of building structures and through-wall surveillance allow intelligence, reconnaissance, and clearance tasks to be performed by driving by or around a building with a small truck. This will greatly improve the security, response time, and covertness of the aforementioned tasks.

In this paper the operational concept for a multifunction radar system capable of imaging and mapping the inside of a building, through-wall tracking of people, and blue-force tracking inside buildings will be discussed. The capabilities are demonstrated with the aid of the SAPPHIRE demonstrator. Some key parameters of SAPPHIRE are listed in Table I; a detailed system description can be found in [1]

Note that an extensive overview of through-wall radar imaging and sensing technologies can be found in [2] and [3] respectively.

TABLE I

SUMMARY OF SAPPHIRE SYSTEM CHARACTERISTICS.

\begin{tabular}{|l|l|}
\hline radar principle & $\begin{array}{l}\text { frequency modulated continuous wave } \\
\text { (FMCW) }\end{array}$ \\
\hline carrier frequency & $2.3 \mathrm{GHz}$ \\
\hline sweep repetition frequency & $250 \mathrm{~Hz}$ \\
\hline polarizations & $\mathrm{VV}, \mathrm{VH}, \mathrm{HV}$, and $\mathrm{HH}$ \\
\hline number or receive channels & 32 in a linear array \\
\hline resolution & $50 \mathrm{~cm}$ in 3D at $10 \mathrm{~m}$ range \\
\hline
\end{tabular}

\author{
R. J. Miller / L. Hyde \\ Advanced Technology Centre, BAE Systems \\ Chelmsford, U.K. \\ robert.j.miller@baesystems.com / \\ lydia.hyde@baesystems.com
}

\section{OPERATIONAL CONCEPT}

The first part of the operational concept is based on a driveby scenario. For stand-off imaging and mapping a linear array is vertically installed on a moving platform. By moving past a building, a 3D measurement grid can be built-up, as is illustrated in Fig. 1. The data obtained with the moving platform can be used to obtain 3D synthetic aperture radar (SAR) images of the (inside of the) building. SAR images provide a first indication of the building layout, but are difficult to interpret visually. Therefore automated extraction of building features is crucial in support of an analyst. Important features are walls, corners, doors and stairwells inside the building. When such features are detected, classified, and located, a building map can be synthesized. The synthesized building map can be projected onto the SAR images aiding visual interpretation.

As part of the mapping task, objects of interest inside the building may be detected and classified. In a disaster situation for example, electrical installations and storerooms with chemicals are of special interest for the rescue workers. In a hostage situation, weapon stashes and explosives must be located. Within the present study, detection and classification of (complex) objects in a building could not be demonstrated.

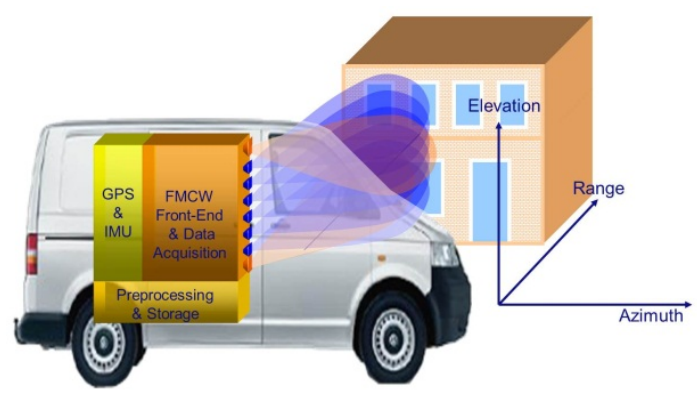

Fig. 1 The drive-by scenario.

In order to further improve situational awareness it is essential to detect humans inside the building and identify them as own personnel, neutrals, or hostiles. This might be achieved by tracking moving people over a relatively long period of time, providing or confirming information about e.g. stairwells 
and doors. Such long-term detection and tracking of moving people constitutes the second part of the operational concept.

For long-term stand-off detecting and tracking of moving people, the radar remains in a stationary position. Now the linear array is rotated by $90^{\circ}$. Rotating the array can be easily and rapidly done in an operational situation. In this horizontal setup the array allows moving people to be tracked with high azimuth resolution, but elevation information can no longer be obtained.

In addition to detection of moving people, localization of motionless people is also of great operational value. In case of a disaster victims may be unconscious and in hostage situations the hostages may be tied. Immobilized people can be located using life-sign detection (heartbeat or breath). In principle, lifesign detection can be performed with the same radar system, when the Doppler sensitivity is sufficient. Within the current study, however, life-sign detection has not been demonstrated.

The third part of the operational concept aims at supporting a rescue team or police squad when they have entered the building. When inside the building it is difficult to maintain one's own position and the whereabouts of other team members. There may be smoke and reception of the Global Positioning System (GPS) may be poor or hostiles may even actively jam GPS. Through-wall blue-force tracking is an important aid in maintaining the overview of the positions of own personnel. For blue-force tracking, the same radar system can be used again if own personnel is equipped with identification (ID) transceivers.

The discussed capabilities, i.e. building imaging, mapping, and (blue-force) tracking, have been demonstrated with the SAPPHIRE radar. The results will be presented in Sections IV through VII. In the following section some considerations on radar propagation through walls will first be given.

\section{THROUGH-WALL RADAR PROPAGATION}

Radar propagation through buildings depends on the building structure and the dielectric properties of the walls. In [4] an extended set of measurements of the dielectric properties of a wide range of materials is presented for a frequency range varying from 2 to $11 \mathrm{GHz}$. Radar waves will not generally pass through the major structural elements of a multi-story building, either because these elements are made of steel or because they are very thick, e.g. reinforced concrete beams.

Concrete used in structural elements is usually reinforced with either a grid of steel reinforcement bars (rebar) or a wire mesh. Mesh or grid spacing may vary from $20 \mathrm{~cm}$ to $50 \mathrm{~cm}$. If the mesh is very closely spaced, this could impede radar propagation substantially. Literature on wire grids indicates that there is no significant attenuation if the radar wavelength is smaller than the mesh spacing [5].

Radar waves will not pass through metal, this includes metal blinds and possibly window coatings containing metal.

\section{BUILDING IMAGING}

In order to demonstrate the first part of the operational concept, 3D measurements were performed on a three-story building. Measurements were made of a row of rooms with different characteristics; a room with closed metal blinds, an empty room divided in two by a glass wall, and a room in use as furniture storage. During the first stage of data analysis the focus was on the empty room on the ground floor. In the room a reference reflector (trihedral corner) was placed on a tripod at $1.5 \mathrm{~m}$ height.

A back-projection algorithm was used to produce a SAR image on a 3D grid with a grid step size (i.e. pixel size) of $25 \mathrm{~cm}$ in all directions. Fig. 2 shows a cross-section at a height of $1.5 \mathrm{~m}$. The range and azimuth resolution are $50 \mathrm{~cm}$. The reflector at a range of $5 \mathrm{~m}$ and azimuth position of $12 \mathrm{~m}$, is clearly visible. Due to the pixel size of $25 \mathrm{~cm}$ the corner reflector yields four pixels. The large reflections between $6 \mathrm{~m}$ and $10 \mathrm{~m}$ in azimuth are due to the closed metal blinds.

In open literature some methods have been reported directly interpreting radar images [6]-[9]. However, from the results shown here, it is clear that reliable (visual) interpretation of SAR images of buildings is difficult, in particular without any knowledge of the actual building layout. Automatic extraction of building features is therefore desired in support of an analyst. Extraction of building features, i.e. building mapping, will be discussed in the following section.

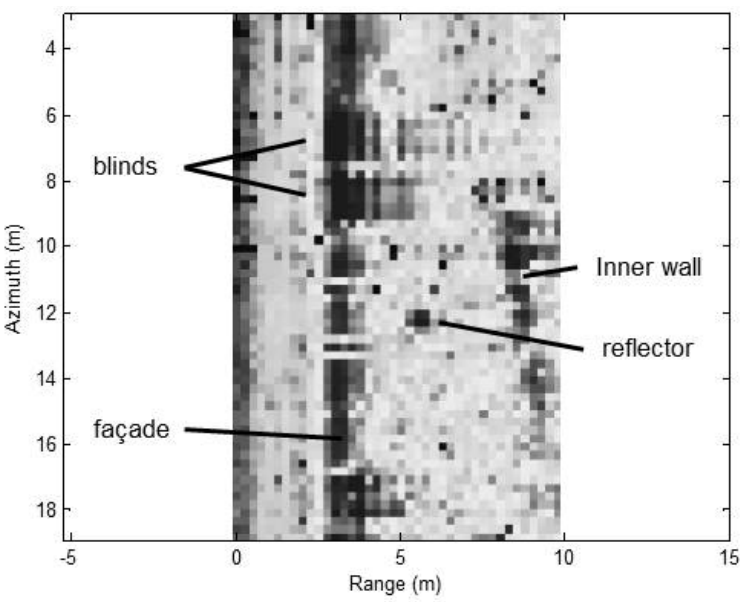

Fig. 2 Radar image of the azimuth/range plane at $1.5 \mathrm{~m}$ height.

\section{BUILDING MAPPING}

Building features may be extracted from the processed SAR images e.g. [10]. However, these methods may be sensitive to multipath reflections and may require two perpendicular radar tracks to image all walls within a building. In literature there is consensus on the fact that building features can be better extracted from the (raw) radar data using model-based techniques, i.e. by classifying and locating canonical scatterers inside a building [11]. Important canonical scatterers inside a building are walls and corners.

\section{A. Building Feature Extraction}

The mapping processing developed for the SAPPHIRE system is indeed based on the detection and location of canonical scatterers. It exploits specific phase relations in the 3D radar 
data induced by different types of scatterers. The phase relations are resolved and the building features are obtained by using sparse reconstruction with an over-complete dictionary. An over-complete dictionary is set of dictionaries (i.e. a set of reference functions). The dictionaries describe the phase references for the different types of canonical scatterers for each point in the reconstruction grid. Three dictionaries are defined to describe the major types of scatterers.

One dictionary defines the phase relation induced by planar walls parallel to the radar (synthetic) aperture. Such walls induce a linear phase relation relative to the aperture. The other two dictionaries describe the phase relation caused by corners. Corners give rise to a quadratic phase relation relative to the aperture. Here distinction is made between forward looking and backward looking phase relations, as is illustrated in Fig. 3. In a building corners may be partly obscured by walls perpendicular to the aperture. In that case, only one half of the parabola may be observable by the radar. It is assumed that a trihedral or T-shaped corner is present when the sparse reconstruction yields a value for both the forward looking and backward looking model at a single point of the reconstruction grid. The definition of the dictionaries and the process of sparse reconstruction are explained in detail in [12].

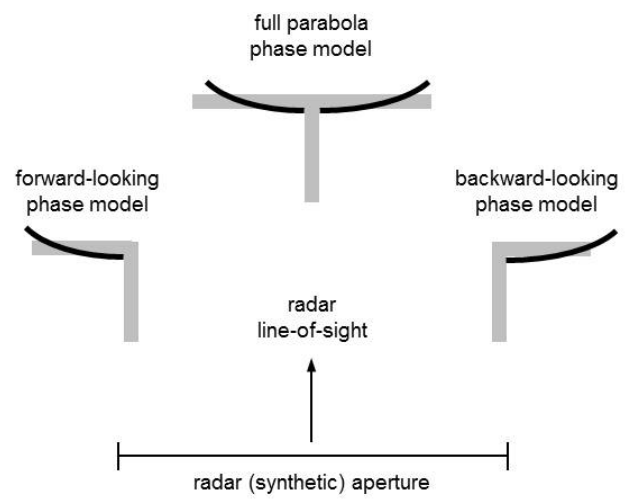

Fig. 3 Phase models (black) for single and double dihedral corners.

For classifying complex objects, such as weapons, phase relations may not be conclusive. Other object characteristics should be incorporated in the model dictionaries. Assuming cylinder-like objects, such as rifles and bazookas, dictionaries tuned to polarimetric behavior may aid classification [13].

It is expected that in future, $2 \mathrm{D}$ and $3 \mathrm{D}$ radar imaging will be based more and more on the use of models to identify and locate canonical scatterers. The conventional point scatterer will be replaced by models for flat surfaces, cylinders, corners, wires and maybe even by models incorporating an entire object of interest, such as models for different types of arms.

\section{B. Building Mapping Results}

The results of the building mapping processing are presented in Fig. 4. The background image is the cross-section already shown in Fig. 2. In this first stage of data analysis, sparse reconstruction has been applied to $2 \mathrm{D}$ data (i.e. after beam forming in elevation and range compression). The reconstruction grid was confined to $8 \mathrm{~m}$ to $15 \mathrm{~m}$ azimuth to exclude the high reflections of the metal blinds. The black lines (-) indicate planar walls and the hooks ( 7 or $\lrcorner$ ) indicate dihedral corners.

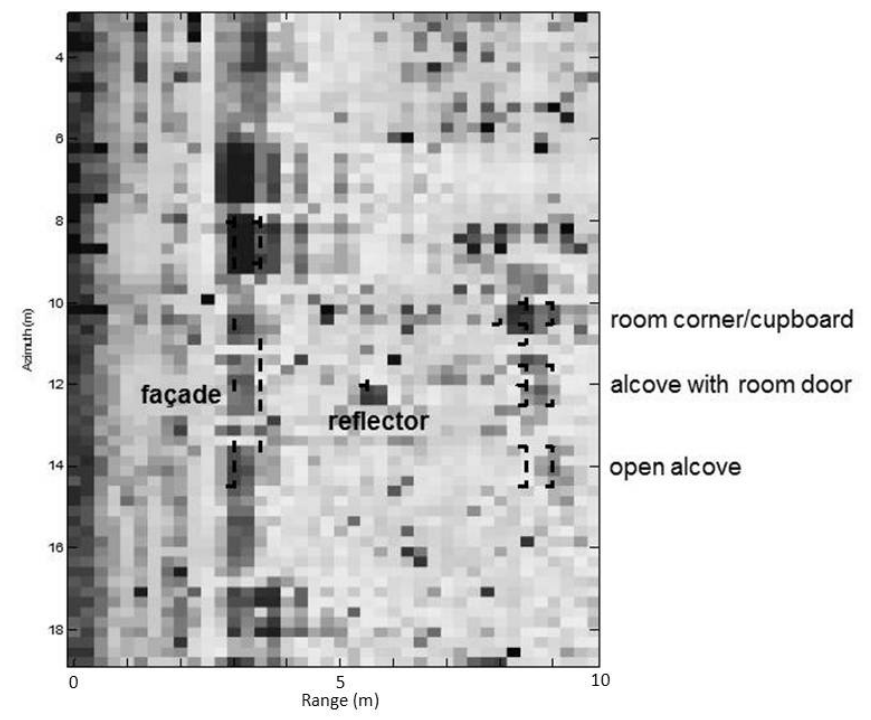

Fig. 4 Result of the building mapping.

The trihedral reflector is correctly classified as a T-shaped corner. Regarding the alcoves and cupboards in the inner building, however, there is confusion about the type of scatterer. The open alcove is correctly classified, but the room door is depicted as a T-shaped corner. Also considering the cupboard in the corner of the room, at $10 \mathrm{~m}$ azimuth, there is confusion. The confusion is probably due to the fact that the depth of the alcoves and cupboards is comparable to the radar resolution. The façade of the building is again reconstructed correctly. The positions of all detected canonical scatterers agree with the ground truth. Note that walls perpendicular to the aperture are not imaged. The location of such walls must be deduced from the presence of the room corners.

\section{Through-Wall Tracking of Moving PeOPle}

The second part of the operational concept has been tested in a bungalow. The façade of the bungalow is formed by large windows on one side and brick walls on the other sides. During the measurements described here, the radar was positioned in front of the windows, see Fig. 5. A camera was placed next to the radar to provide ground truth (as far as allowed by the inner building structure).

\section{A. Tracker Implementation}

Through-wall tracking of people is an important topic and different approaches have been described in literature, e.g. [14], [15]. The tracker implementation used in the present study is briefly discussed in this section.

The first step in the tracker is the generation of 2D radar images by applying a back-projection algorithm to the data (no sparse reconstruction). These 2D images are used as input for Doppler processing; to each pixel a Fourier transform is applied over time resulting in a $3 \mathrm{D}$ data set with dimensions $\{x$, $\left.y, v_{d}\right\}$. The integration time is $0.2 \mathrm{~s}$ such that the response of a 
moving person remains in a single cell. In order to make the motion of the persons well behaved there is a $50 \%$ overlap between successive Doppler integration times.

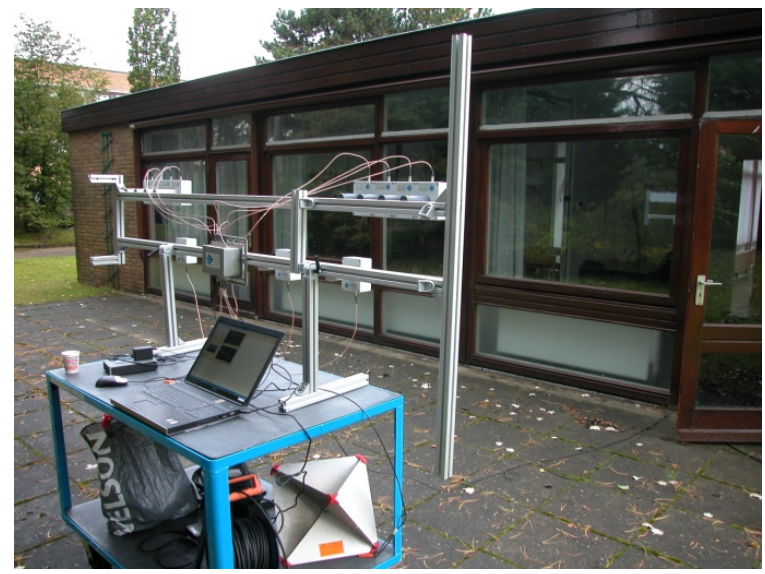

Fig. 5 The SAPPHIRE radar system in front of the bungalow.

At the beginning of each measurement a few seconds without any person present was used to get a background estimate in $\left\{x, y, v_{d}\right\}$. This background estimate was used to set the detection threshold. Due to the large influence of a person on its surroundings the detection threshold needs to be relatively high.

The detections are clustered in 3D with allowed gaps of two cells in all three directions. Only clusters with at least five detections are considered. Smaller clusters are removed. The center of gravity for each dimension $\left\{x, y, v_{d}\right\}$ is obtained based on a weighted sum of the pixel values in the cluster. The weight is given by the power of the pixel. The remaining clusters are fed to the tracker.

Tracking of a moving person is difficult due to possible erratic behavior, i.e. sudden changes in direction or speed. Due to the relatively high update rate and expected low velocities, the assumption has been made that the moving objects have zero velocity. The predicted position is thus taken as the last position and the association rule is based on the distance between the current cluster of detections and the existing track and the difference between the associated Doppler velocities: the 2D spatial distance should be less than $1 \mathrm{~m}$, and the Doppler velocity difference should be less than $0.5 \mathrm{~m} / \mathrm{s}$. The new position is obtained with an alpha-filter, more explicitly the mid-point of the predicted position and the actual cluster position.

\section{B. Results of Radar Tracking of Moving People}

An example of the output of the tracker algorithm is shown in Fig. 6. The left image shows the response of stationary objects, defined as clusters within five Doppler bins around zero Doppler. The middle image shows the response of moving objects, defined as clusters in all other Doppler bins. The right image shows two active tracks associated with two people walking around. In all results shown the radar system is located at the origin.

From the radar tracking results the following was noted. Tracking a person moving radially is well behaved. Due to the rapid motion the response competes with less clutter (i.e. sidelobes from stationary objects such as walls). However, when the person is stationary the response disappears in the stationary clutter. Techniques were applied to remove this assumed stationary clutter, but due to the interaction between persons and the environment the clutter response changes (multipath from person to walls and ceilings for instance). It was therefore not possible to maintain the track while a person remained stationary although in one example a sitting person making a telephone call moved sufficiently to maintain the track.
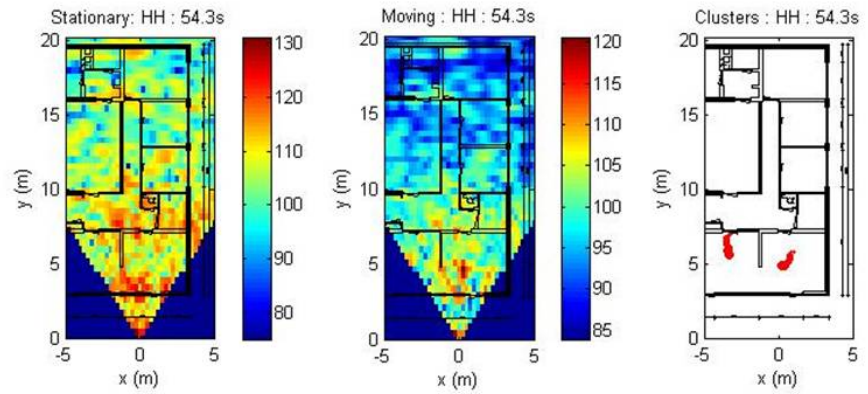

Fig. 6 The output of the tracking algorithm; response of stationary objects (left), response of moving objects (middle), and radar tracks associated with two moving people (right)

Monitoring of the tracks over longer periods of time allows refinement of the building layout. In Fig. 7 all tracks with at least ten associated clusters are plotted on top of the building layout. The combination of the track history and the floor plan indicates that the door located at $x \approx 0 \mathrm{~m}$ and $y \approx 7 \mathrm{~m}$ is in regular use. The door at $x \approx-2.5 \mathrm{~m}$ and $y \approx 7 \mathrm{~m}$ is also in use. The track history can thus indicate possible position of doors that are in use (and stairs when 3D processing is used). In particular for hostage scenarios it is important to know not only the position but also the routes of all persons present. Doors may be blocked, thus indication of doors in use is of vital importance for planning ingression routes for a rescue squad.
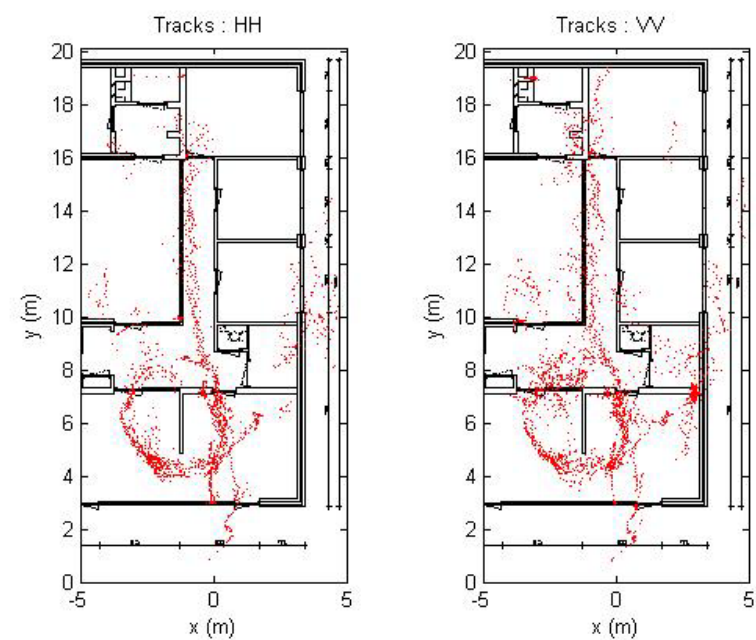

Fig. 7 The results of through-wall tracking of moving people.

In Fig. 7, multipath effects are visible, e.g. the cluster in the corner of the room at $x \approx 3 \mathrm{~m}$ and $y \approx 7 \mathrm{~m}$ in the right image. 
By way of illustration, the cluster selection criterion was lowered to a minimum of four detections. In Fig. 8 the clusters associated with persistent tracks, i.e. tracks with more than 60 successive clusters associated, are shown. Four regions have been indicated that clearly yield tracks that could not be associated with a person at that position. Region 2 can be associated with the large reflection from the window and the radar operator seems to be detected at the origin. The clusters in Region 4 seem to indicate a direct reflection. The distance at which these reflections are shown is the distance of the (in this case) wall. The clusters in Regions 1 and 3 are almost always present when a person is walking in the area in between these regions toward the radar. These are multipath effects that can be identified when studying the track history.

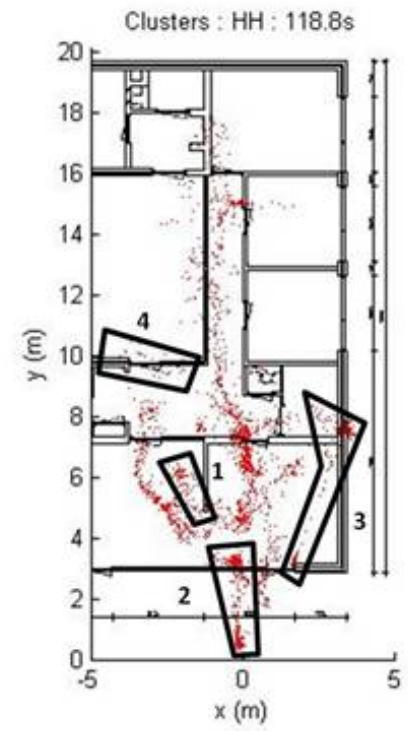

Fig. 8 Clusters associated with persistent tracks. The clusters inside the designated regions could not be associated with the tracks of moving people.

\section{Through-WALl Blue-ForCE TRACKING}

When own personnel are inside the building, it is desired to track and identify them as such. This is possible by equipping friendly forces with ID transceivers. If the transceiver waveform is matched to the radar waveform, the same radar system can be used for blue-force tracking as for mapping and tracking. Own personnel can be located and identified by interrogation of the ID transceivers. The transceivers reply with a unique identity tag and if possible their GPS position. When GPS is not available, locating friendly forces is still possible by ranging and direction finding of the transceiver replies.

Experiments have been performed to demonstrate the feasibility of transceiver detection and direction finding. These experiments were carried out using the same measurement geometry as for the moving people tracking measurements. However, a different radar antenna system setup had to be used, as is explained hereafter.

\section{A. Transponder Operation}

Within the current project a (simple) radar transponder was used to demonstrate through-wall blue-force tracking. This transponder is trigger-locked to the radar system and transmits the same waveform. During the experiments, the transponder was triggered by the radar using a long cable between the transponder and the radar, such that the transponder hardware could be kept simple.

The use of a trigger cable had one major disadvantage. The SAPPHIRE linear array is synthesized by using the multiple input multiple output (MIMO) technique. The antenna comprises four transmitters and eight receivers, see Fig. 9 top, resulting in a fully-filled linear MIMO array of 32 receive channels. However, using the trigger cable, the different transmitter-receiver paths are no longer distinguishable. The trigger signal triggers one of the four transmitters and at the same time it triggers the transponder, but with constant delay corresponding to the cable length and not to the actual range of the transponder. Consequently, the receivers get a phase shift due to the different paths from the transponder to each receiver. The distance and angular measurements are thus based on the one-way phase shift: the coherent monostatic MIMO concept is no longer applicable. The blue-force tracking measurements were therefore carried out using the 2D array setup shown in Fig. 9 (bottom). The receive elements are spaced half a wavelength and one-way near-field focusing techniques can be applied. The angular accuracy is however reduced by a factor of four due to the shorter array length.
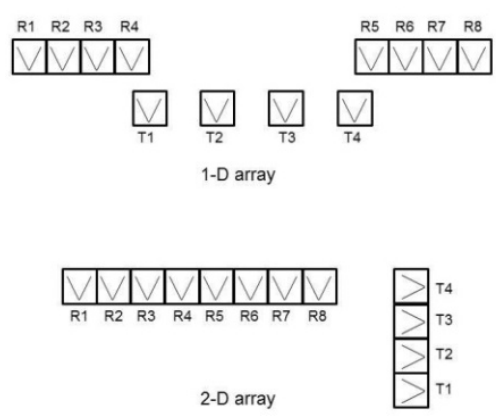

Fig. 9 SAPPHIRE MIMO array layout; T1 to T4 indicate the four transmitters $\mathrm{R} 1$ to $\mathrm{R} 8$ indicate the eight receivers. The top figure shows the linear array layout as used for imaging, mapping and people tracking; the bottom figure shows the 2D array layout as used for blue-force tracking.

The discussed hardware solution is adequate to demonstrate transponder detection and location in through-wall scenarios. For operational use it is mandatory to develop more complex, wireless transceivers that preserve the two-way phase shift.

\section{B. Results of Blue-Force Tracking}

In Fig. 10, two near-field focused images of transponder responses are presented in the two left images. By averaging these responses, the right image is obtained. Here the black dot designates the average position. This averaged position can be used as input for the transponder tracker, which can be combined with the radar tracker described in the last section.

In Fig. 11, a result is shown of tracking a person carrying the transponder around the building. The left image shows the averaged response of the transponder, the middle image shows the radar response of moving objects, and the right image 
shows the transponder track (indicated by the black dot) and the radar track of the person carrying the transponder (designated by the red dots superimposed on the transponder track). Since in this case, the transponder and radar tracks can be associated, i.e. the plots overlap, the radar track can be identified as a blue-force track.
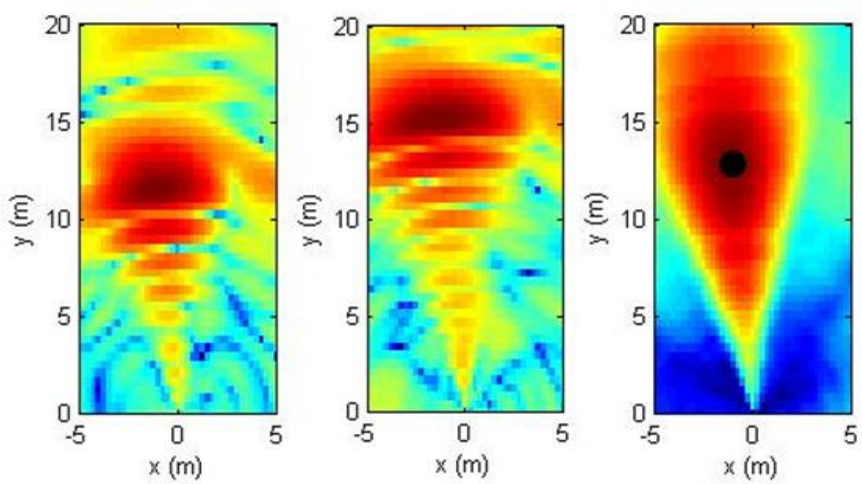

Fig. 10 Two responses of the transponder (two left images) and the associated averaged response (right image). The averaged plot (the black dot in the right image) can be fed to a tracker.

In the measurement shown in Fig. 11 the person carried the transponder from the top right room through the hall to the living room at near range. It is noticeable that the radar response of the person was not detected when the person was in the top right room. The transponder response, however, could be detected and located.

The results obtained with this simple transponder show the potential to track own personnel through several walls and identify radar tracks as blue-force tracks.
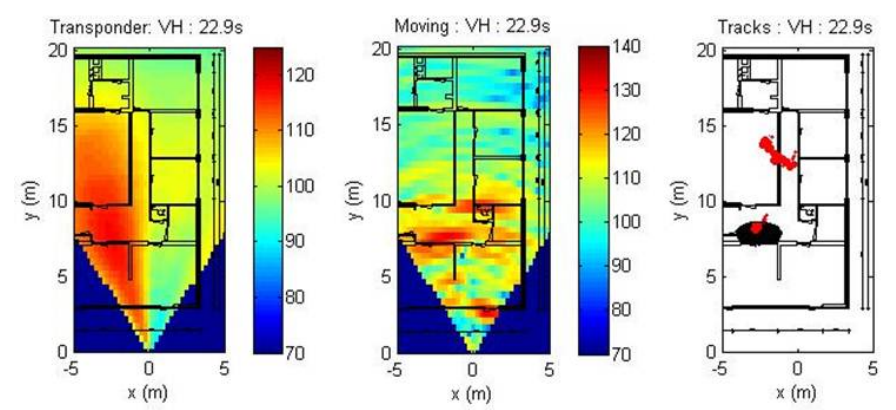

Fig. 11 The left image shows the averaged response of the transponder, the middle image shows the radar response of moving objects, and the right image shows the associated transponder (black dot) and radar tracks (red dots).

\section{CONCLUSION}

In this paper the operational concept for a multifunction radar for through-wall surveillance has been described. The operational concept focuses on a single radar system capable of (covert and stand-off) building imaging and mapping, detection of stationary objects and immobilized persons in a building, detection and tracking of moving people, and blue-force tracking. The imaging, mapping, and tracking capabilities have been demonstrated with representative measurements.
Future work will be on continues improving the mapping capabilities and investigation of novel waveforms for multipath and clutter suppression.

\section{ACKNOWLEDGMENT}

This work was supported by the European Defence Agency under contract number EDA Contract 11.R\&T.OP.131 "Radar Technology for Inside Building Awareness (RIBA)." The findings of this contract are EDA property. IPR reserved.

\section{REFERENCES}

[1] F. M. A Smits, J. J. M. de Wit, W. L. van Rossum, A. P. M. Maas, R. J. Bolt, and C. M. Lievers, "3D Mapping of Buildings with SAPPHIRE," in Proc. EMRS-DTC Technical Conf., Edinburgh, U.K., July 7-8, 2009.

[2] M. G. Amin, Through-the-Wall Radar Imaging, Boca Raton: CRC Press, 2010.

[3] "Sensing-Through-The-Wall Technologies", Task Group SET-100, NATO RTO, Tech. Rep. AC/323(SET-100)TP/360, October 2011.

[4] A. Muqaibel, A. Safaai-Jazi, A. Bayram, A. M. Attiya, and S. M. Riad, "Ultrawideband through-the-wall propagation", IEE Proc. Microw. Antennas Propag., vol. 152, no. 6, pp. 581-588, December 2005.

[5] J. R. Wait, "Reflection at arbitrary incidence from a parallel wire grid," Applied Scientific Research, Section B, vol. 4, pp. 393-400, 1955.

[6] "Building / Wall Penetration Radar O PEN," SRC, Product Brochure. Available online: http://www.srcinc.com/

[7] C. Le, T. Dogaru, L. Nguyen, and M. A. Ressler, "Ultrawideband (UWB) Radar Imaging of Building Interior: Measurements and Predictions," IEEE Trans. Geosci Remote Sensing, vol. 47, no. 5, pp. 1409-1420, May 2009.

[8] P. Sévigny, D. J. DiFilippo, T. Laneve, B. Chan, J. Fournier, S. Roy, B. Ricard, and J. Maheux, "Concept or Operation and Preliminary Experimental Results of the DRDC Through-Wall SAR System," Proc. SPIE, vol. 7669, April 2010.

[9] W. Zhang, A. Hoorfar, and C. Thajudeen, "Building Layout and Interior Target Imaging with SAR Using an Efficient Beamformer," in Proc. IEEE Int. Symp. Antennas Propagation, Spokane, U.S.A., July 3-8, 2011, pp. 2087-2090.

[10] M. Aftanas and M. Drutarovsky, "Imaging of the Building Contours with Through the Wall UWB Radar System," J. Radioengineering, no. 3, vol. 18, pp. 258-264, September 2009.

[11] E. J. Baranoski, "Through Wall Imaging: Historical Perspective and Future Directions," J. Franklin Institute, vol. 345 pp. 556-569, Jan. 2008.

[12] J. J. M. de Wit, L. Anitori, W. L. van Rossum, and R. G. Tan. "Radar Mapping of Buildings using Sparse Reconstruction with an Overcomplete Dictionary," in Proc. EuRAD, Manchester, U.K., Oct. 13-14, 2011, pp. 9-12.

[13] T. Dogaru and L. Calvi, "Through-the-Wall Small Weapon Detection Based on Polarimetric Radar Techniques," Army Research Laboratory, Tech. Rep. ARL-TR-5041, December 2009. Available online: http://www.dtic.mil/cgi-bin/GetTRDoc?AD=ADA510201

[14] X. P. Masbernat, M. G. Amin, F. Ahmad, and . Ioana, "An MIMO-MTI Approach for Through-the-Wall Radar imaging Applications," in Proc. WDD, Niagara Falls, Canada, Aug. 8-13, 2010.

[15] A. Buonanno, M. D’Urso, M. Felaco, G. Prisco, L. Angrisani, and R. Schiano Lo Moreillo, "Through-the-Wall Tracking of Moving Targets: Tests on Experimental Data," in Proc. IEEE Radar Conf., Kansas City, U.S.A., May 23-27, 2011. 